\title{
Caracterização da Taxa de Absorção Específica do Celular na Cabeça Humana, Usando o Método LN-FDTD
}

\author{
Adelson B. Medeiros, Vanilson G. Pereira, Brigida R.P. da Rocha
}

\begin{abstract}
Neste trabalho será abordado o estudo da absorção de energia eletromagnética em tecidos biológicos da cabeça humana, proveniente de aparelhos celular na faixa de $900 \mathrm{MHz}$ e 1800MHz.O desenvolvimento de um programa para o cálculo da Taxa de Absorção Específica (SAR) na cabeça do usuário de Telefone Celular. È apresentado a implementação do método Diferenças Finitas no Domínio do Tempo não Ortogonal Local (LN-FDTD), e a utilização de Meios Uniaxiais Perfeitamente Casados (UPML). Comparando os resultados Obtidos com os da literatura.
\end{abstract}

Keywords-Telefone Celular, Efeitos Biológicos, Taxa de Absorção Específica (SAR), Diferenças Finitas no Domínio do Tempo não Ortogonal Local (LN-FDTD), Meios Uniaxiais Perfeitamente Casados (UPML), Radiação não-ionizante .

\section{INTRODUÇÃO}

A popularidade da telefonia móvel no mundo globalizado, fez com que ocorresse uma demanda crescente das necessidades das pessoas na sociedade globalizadas em se mobilizar no sentido de diminuir as distâncias entre elas, o que veio a facilitar a comunicação móvel e ao mesmo tempo proporcionar novas perspectivas de vida. Mesmo com todo este avanço que tem se dado na telefonia móvel, através de seus benefícios e aplicações em prol da sociedade, este tipo de sistema acarreta danos à saúde humana, uma vez que em diversas situações estes aparelhos podem emitir potências elevadas quando estão muito próximas à cabeça do usuário. Nestes casos, devido a grande exposição das radiações eletromagnéticas as pessoas ficam submetidas, a uma parcela importante da energia irradiada, é absorvida em tecidos da cabeça: pele, gordura, osso e cérebro etc, alguns dos quais são formados de elementos muitos sensíveis e de extrema importância para o ser humano, como por exemplo: os neurônios, cromossomos etc. Esta absorção de energia eletromagnética não ionizante pode provocar fundamentalmente dois efeitos: (a) Os "Efeitos Térmicos"e (b) "Efeitos não Térmicos"[1][2]. Os efeitos térmicos são conhecidos pelo menos há cinqüenta anos(50 anos). Decorrem do aquecimento do tecido. A radiação é não somente absorvida ao nível da pele, mas também em níveis mais profundo do corpo, causando um aumento de temperatura não percebido pelos sensores térmicos naturais, já que localizados superficialmente. Esse calor gerado internamente a depender do tempo de exposição da intensidade do campo e da espessura do tecido, não pode ser compensado e ocasiona graves danos:(a) aumento da temperatura nos órgãos, (b) alterações no funcionamento elétrico dos mesmos, devido a modificações no funcionamento químico das células e (c) alterações no mecanismo de transmissão de sinais elétrico, do cérebro para os músculos, segundo Salles[3]. Os limites de exposição são variáveis com a freqüência, em parte por considerar a ressonância e também por considerar a menor profundidade de penetração dos campos eletromagnéticos em freqüências mais elevadas. A ressonância é importante, pois quando este fenômeno ocorre, resulta em maior absorção de energia, por exemplo: a cabeça de um adulto pode apresentar ressonância na faixa de 400 a $500 \mathrm{MHz}$, e na cabeça de uma criança a ressonância pode ocorrer na faixa de 700 a $800 \mathrm{MHz}[8]$.Os efeitos não térmicos são efeitos bioquímicos ou eletro-físico causados diretamente pelos campos eletromagnéticos induzidos. Estes efeitos ainda fazem parte de estudos o qual apresenta resultados conflitantes na literatura científica internacional. Os efeitos não térmicos demonstrados na literatura incluem alteração no fluxo de íons através das membranas das células (afetando particularmente as propriedades eletro-fisiológicos das células nervosas) alterações na síntese de DNA e na transcrição de RNA, incluindo hormônios, neurotransmissores e fatores de crescimento. Alterações no fluxo de cálcio em células, na barreira cérebro-sangue (blood-brain barrier) que protege o cérebro de certas toxinas e no desenvolvimento de tumores cerebrais que foram reportados por[1]. No problema que se estar propondo, utiliza-se a radiação não-ionizante para a determinação da taxa de absorção específica do celular na cabeça humana, fazendo variar a distância do celular a cabeça e a freqüência do celular. Utilizamos o método das diferenças finitas no domínio do tempo não-ortogonal local (LN-FDTD)[4], que contribui no desenvolvimento de um algoritmo para obtenção da (SAR) do celular na cabeça do usuário. A principal contribuição deste trabalho é apresentar um método numérico que resolve o problema de staircase em uma superfície "Elipsóide"e a obtenção dos resultados que são mais precisos, e ganho no tempo computacional. O restante deste trabalho é organizado como segue. Seção II fornece a implementação teórica do método LN-FDTD. Seção III apresenta a descrição do modelo. Seção IV Descrição do modelo LN-FDTD da cabeça humana. Seção V. Resultados do modelo da cabeça humana. Seção VI. Conclusão. 


\section{IMPLEMENTAÇÃO TEÓRICA DO MÉTODO LN-FDTD}

Definiu-se uma grade estruturada irregular não ortogonal sendo composto de duas malhas denominadas de primária e secundárias. A malha primária é projetada para adaptar-se a geometria do problema, a que pode ser realizado mediante o uso de um gerador de malhas adaptativas. Esta malha pode também ser escolhida para se alinhar com as componentes do campo elétrico ou magnético, dependendo da condição de contorno que é imposta na geometria física. Então, a segunda malha é gerada pela conexão dos baricentros das células da malha primária, como mostra a figura (1).

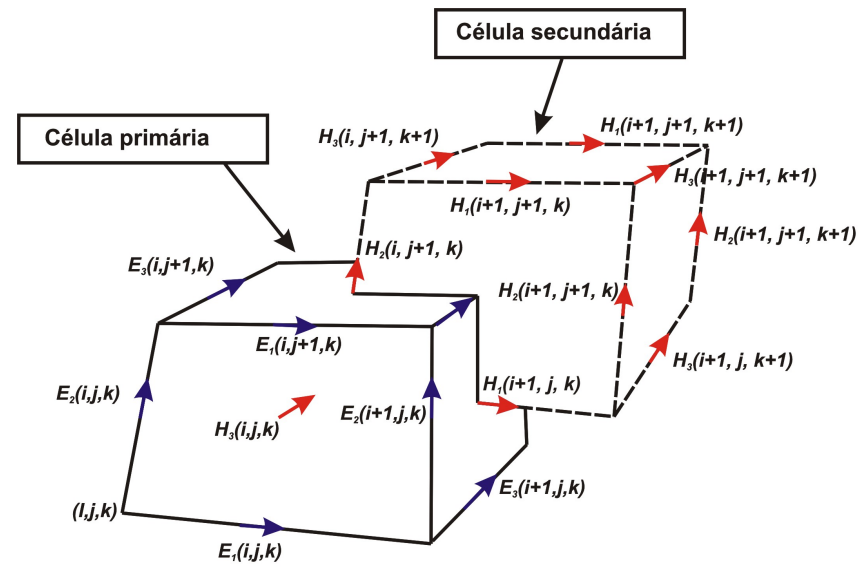

Fig. 1

CÉLULA PRIMÁRIA E SECUNDÁRIA DE UMA MALHA ESTRUTURADA IRREGULAR NÃO ORTOGONAL

Para as geometrias que possam ser discretizadas por este tipo de grade, define-se os vetores unitários de base, $A_{i}(1,2,3)$, pelas arestas das células das malhas primárias e secundária, conforme mostra a figura (2).

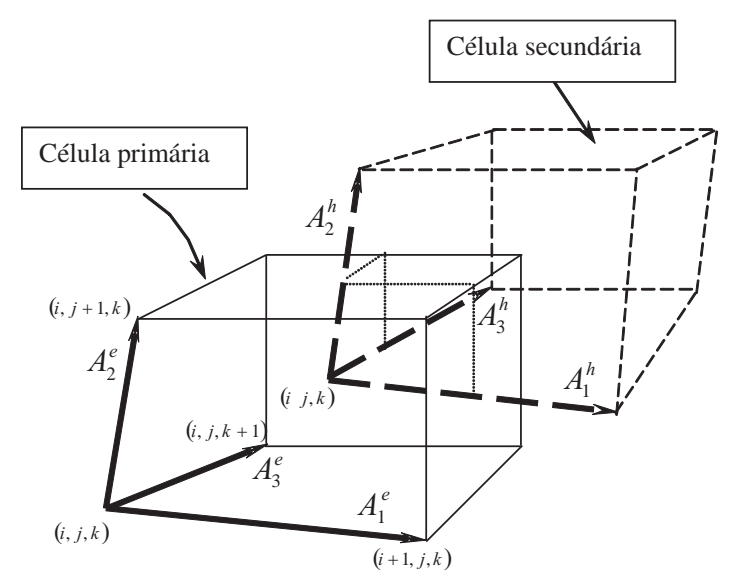

Fig. 2

DEFINIÇão DOS VETORES UNITÁRIOS DE BASE PARA AS CÉLULAS PRIMÁRIA E SECUNDÁRIA NO SISTEMA DE COORDENAS CURVILÍNEAS LOCAIS
As equações de Maxwell na forma integral por serem naturalmente aplicadas em um espaço não ortogonal irregular serão utilizadas para obtenção das componentes de atualização dos campos elétricos e magnéticos no domínio do tempo, usando-se o método LN-FDTD. Considerando-se um meio isotrópico e livre de fontes[5], pode-se escrever:

$$
-\frac{\partial}{\partial_{t}} \int_{\Omega} \mu H \cdot d a=\oint_{\partial \Omega} \mathrm{E} \cdot \mathrm{dl}
$$

$\mathrm{e}$

$$
\frac{\partial}{\partial t} \int_{\Omega} \epsilon E \cdot d a=\oint_{\partial \Omega} \mathrm{H} \cdot \mathrm{dl}
$$

O campo elétrico $\mathrm{E}(\mathrm{i}, \mathrm{j}, \mathrm{k})$ em um ponto $(\mathrm{i}, \mathrm{j}, \mathrm{k})$ da grade, que pode ser expresso em termos das duas bases, respectivamente, como,

$$
\begin{aligned}
& E(i, j, k)=\sum_{j=1}^{3} E^{j}(i, j, k) A_{j}(i, j, k) \\
& E(i, j, k)=\sum_{j=1}^{3} E_{j}(i, j, k) A^{j}(i, j, k)
\end{aligned}
$$

Em (3) e (4), os coeficientes $E^{i}$ e $E_{i}$ são chamados de componentes contravariantes e covariantes, respectivamente, do campo elétrico. A componente covariante $E_{i}(i, j, k)$ representa o fluxo de $E(i, j, k)$ ao longo da aresta i, e $E^{i}(i, j, k)$ representa o fluxo total do campo elétrico passando através da face $i$.

A partir da interpretação física, podem-se escrever as expressões para os campos (E e H), que aparecem na integral de linha (lado direito) das equações (11) e (2). Resolve-se a (1), pelo método LN-FDTD, para a componente contravariante do campo magnético $H^{i}(i, j, k)^{n+\frac{1}{2}}$, assumindo-se que a mesma seja constante sobre a face de cada célula, e escrita em função do volume da célula, resultando em,

$$
\begin{aligned}
\mu \frac{\partial}{\partial_{t}} H^{1}(i, j, k)^{n} V= & {\left[E_{3}(i, j+1, k)^{n}-E_{3}(i, j, k)^{n}\right] } \\
& -\left[E_{2}(i, j, k+1)^{n}-E_{2}(i, j, k)^{n}\right]
\end{aligned}
$$

No sistema de coordenadas curvilíneas locais, o volume $V$ é definido para cada componente contravariante, a partir dos vetores de base unitária que definem a célula na figura (2).

Para o cálculo do volume relativo a uma dada face, é necessário conhecer o vetor unitário de base da componente contravariante normal a esta face e os quatro vetores de base unitária, da malha dual, que definem a face através da qual o campo vetorial contravariante passa. Desta forma, o volume para a componente $H^{1}$, de acordo com as figuras (1) e 2) é dado por:

$$
V_{1}^{h}=A_{1}^{h}(i, j, k) \bullet\left\{\begin{array}{c}
\left(\frac{A_{2}^{e}(i, j, k+1)+A_{2}^{e}(i, j, k)}{2}\right) \bullet \\
\bullet\left(\frac{A_{3}^{e}(i, j+1, k)+A_{3}^{e}(i, j, k)}{2}\right)
\end{array}\right\}
$$


Obtêm-se a expressão de atualização para a componente contravariante do campo magnético $H^{1}(i, j, k)^{n+\frac{1}{2}}$, o que é feito substituindo (6) em (5) resulta em,

$$
\begin{aligned}
& H^{1}(i, j, k)^{n+\frac{1}{2}}=H^{1}(i, j, k)^{n-\frac{1}{2}}-\frac{\Delta t}{\mu V_{1}^{h}}(i, j, k) \bullet \\
& \text { - }\left\{\begin{array}{l}
\left(E_{3}(i, j+1, k)^{n}-E_{3}(i, j, k)^{n}\right)- \\
\left(E_{2}(i, j, k+1)^{n}-E_{2}(i, j, k)^{n}\right)
\end{array}\right\}
\end{aligned}
$$

As outras componentes contravariantes do campo magnético $H^{2}(i, j, k)^{n+\frac{1}{2}}$ e $H^{3}(i, j, k)^{n+\frac{1}{2}}$, e os vetores $V_{2}^{h}$ e $V_{3}^{h}$ são obtidos de maneira análoga. A solução da equação (2), pelo método LN-FDTD, para calcular a componente contravariante do campo elétrico $E^{1}(i, j, k)^{n+1}$.

$$
\begin{aligned}
& E^{1}(i, j, k)^{n+1}=E^{1}(i, j, k)^{n}+\frac{\Delta t}{\epsilon(i, j, k) V_{1}^{e}(i, j, k)} \bullet \\
& \text { • }\left\{\begin{array}{c}
\left(H_{3}(i, j, k)^{n+\frac{1}{2}}-H_{3}(i, j-1, k)^{n+\frac{1}{2}}\right)- \\
\left(H_{2}(i, j, k)^{n+\frac{1}{2}}-H_{2}(i, j, k-1)^{n+\frac{1}{2}}\right)
\end{array}\right\}
\end{aligned}
$$

De modo análogo, obtêm-se as componentes contravariantes do campo elétrico $E^{2}(i, j, k)^{n+\frac{1}{2}}$ e $E^{3}(i, j, k)^{n+\frac{1}{2}}$, com os seus respectivos volumes dados por:

$$
V_{1}^{e}=A_{1}^{e}(i, j, k) \bullet\left\{\begin{array}{c}
\left(\frac{A_{2}^{h}(i, j, k)+A_{2}^{h}(i, j, k-1)}{2}\right) \bullet \\
\bullet\left(\frac{A_{3}^{h}(i, j, k)+A_{3}^{h}(i, j-1, k)}{2}\right)
\end{array}\right\}
$$

De maneira análoga obtêm-se os volumes $V_{2}^{e}$ e $V_{3}^{e}$. É necessário converter as componentes contravariantes do campo elétrico $E$ em componentes covariantes, para que as componentes contravariantes do campo $H$ possam ser calculadas, e vice-versa.

\section{DESCRIÇÃO DO MODELO}

O desenvolvimento deste trabalho se dará na UPML[6], cujas paredes são revestidas de camadas absorventes (ABC), o qual desempenha o papel das condições de contorno. O interior da UPML é chamado de região de análise, e será colocada uma cabeça humana com as suas respectivas propriedades dielétricas: condutividade, permeabilidade e permissividade de cada tecido, afastada a $9 \mathrm{~mm}$ do dipolo de freqüência variável de $900 \mathrm{MHz}$, que será medido campo elétrico médio em cada ponto da célula, de acordo com a figura (3).

\section{DESCRIÇÃO DO MODELO LN-FDTD DA CABEÇA HUMANA}

Neste trabalho faz-se uma descrição dos dados e o desenvolvimento do modelo numérico LN-FDTD. O modelo é detalhado com os diferentes tipos de tecidos: pele, gordura, couro-cabeludo, osso, sangue, etc., e com as suas respectivas propriedades dielétricas. O modelo mencionado acima, é definido com as seguintes dimensões: $264 \mathrm{~mm}$ de altura, 177 $\mathrm{mm}$ de largura e $240 \mathrm{~mm}$ de profundidade. Foram obtidas a

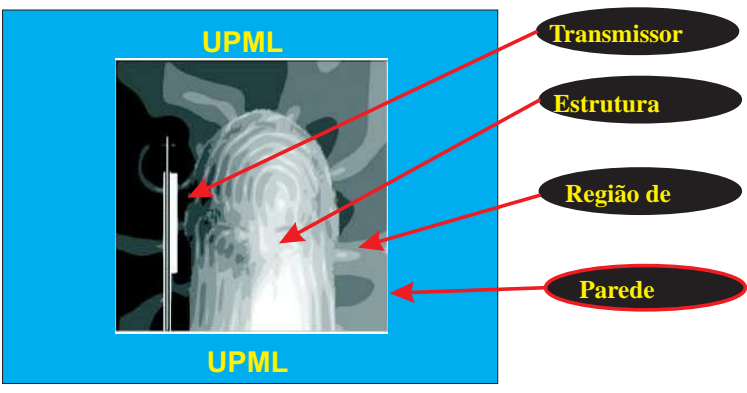

Fig. 3

MODELO DE UPML PARA IMPLEMENTAÇÃO DA TÉCNICA LN-FDTD

partir de imagens médicas disponíveis. No total foram usadas 226 imagens de cortes axiais, com espaçamento de $9 \mathrm{~mm}$, cedidas pelo Hospital "Ofir Loyola", e recomendado pelo laboratório de pesquisa AT Telstra Research Laboratories (TLR)[7] cada imagem é composta de 177 x 240 pontos ou pixeis, compondo uma malha de 42.480 células. Para auxiliar na discretização dos tecidos, foram usadas imagens de tomografia computadorizada e ressonância magnética. Observa-se que o pico do SAR está na base do nariz. Este pico coincide também com a presença dos tecidos mais elevados da condutividade: pele, sangue, e cartilagem, de acordo com os valores da tabela (I)[7].

TABELA I

Propriedades Dielétricas dos Tecidos da CabeÇa Humana

\begin{tabular}{|c||c|c|c|}
\hline Tecido & $\begin{array}{c}\text { Condutividade } \\
\left(\Omega^{-1} m^{-1}\right)\end{array}$ & $\begin{array}{c}\text { Permissividade } \\
\text { Relativa }\end{array}$ & $\begin{array}{c}\text { Densidade de } \\
\text { massa }\left(\mathrm{Kg} / \mathrm{m}^{3}\right)\end{array}$ \\
\hline cartilagem & 0.782 & 42.65 & 1000 \\
\hline musculo & 0.969 & 55.95 & 1050 \\
\hline olho & 1.900 & 70.00 & 1000 \\
\hline cérebro e nervos & 0.766 & 45.80 & 1050 \\
\hline pele & 0.867 & 41.40 & 1000 \\
\hline sangue & 1.180 & 62.00 & 1000 \\
\hline crânio & 0.242 & 16.62 & 1850 \\
\hline
\end{tabular}

\section{RESULTADOS DO MODELO DA CABEÇA HUMANA}

Estudam-se os efeitos da radiação eletromagnética não ionizante na cabeça humana; como a distribuição de campo elétrico e a SAR. Inicialmente, realizaram-se simulações para o problema consistindo de uma onda plana uniforme interagindo com a cabeça humana. Adotamos para uma simulação experimental, um meio não homogêneo, com as constantes dielétricas da tabela (I), e , para às frequiências de $900 \mathrm{MHz}$ e $1800 \mathrm{MHz}$ com uma potência de $1 \mathrm{~W}, 0.6 \mathrm{~W} 0.25 \mathrm{~W}$, que foram recomendados pelo IEEESCC-34/S-2. Para um modelo simples de cabeça humana em 2-D com um corte sagital no plano xy, excitado por uma antena dipolo de comprimento de $90 \mathrm{~mm}$. A variação da distância, entre à antena e a cabeça; $9 \mathrm{~mm}$ orientado ao longo do eixo z. Os resultados adquiridos referentes a simulação de campo elétrico e SAR, após 1750 interações, de acordo com a tabela (II). 
TABELA II

\begin{tabular}{|c|c|c|c|c|}
\hline $\begin{array}{c}\text { Frequência } \\
\text { de } \\
\text { operação }\end{array}$ & $\begin{array}{l}\text { Distância } \\
(\mathrm{mm})\end{array}$ & $\begin{array}{c}\text { Potência } \\
\text { Saida } \\
\text { Antena(W) }\end{array}$ & $\begin{array}{c}\text { Campo Elétrico } \\
\text { Médio(V/m) }\end{array}$ & $\begin{array}{c}\text { SAR } \\
\text { Médio } \\
(\mathrm{W} / \mathrm{Kg})\end{array}$ \\
\hline $900 \mathrm{MHz}$ & 9 & $\begin{array}{c}1.0 \\
0.6 \\
0.25\end{array}$ & $\begin{array}{r}126 \\
75.6 \\
31.5\end{array}$ & $\begin{array}{l}7.73 \\
2.78 \\
0.48\end{array}$ \\
\hline $1800 \mathrm{MHz}$ & 9 & $\begin{array}{c}1.0 \\
0.6 \\
0.25\end{array}$ & $\begin{array}{c}165 \\
106.5 \\
44.4\end{array}$ & $\begin{array}{c}18.9 \\
7.9 \\
1.3\end{array}$ \\
\hline
\end{tabular}

Baseando-se nos valores da tabela (II), constroem-se os gráficos SAR versus distância para as faixas de freqüência de $900 \mathrm{MHz}$ e $1800 \mathrm{MHz}$, na qual os gráficos das figuras (4) e (5) apresentam uma maior compreensão dos dados simulados. Podem-se observar que os resultados adquiridos em relação as potências de $1 \mathrm{~W}$ e $0.6 \mathrm{~W}$, nas frequências acima citadas, mostram que os valores da SAR estão acima dos valores previsto por lei. Enquanto que os dados relativos a potência de $0.25 \mathrm{~W}$ apresentado na tabela (II), mostram que os valores da SAR estão dentro dos limites estabelecidos pelas agências internacionais como a ICNIRP[8] e a própria agência nacional ANATEL [9], que é estipulado em $2 \mathrm{~W} / \mathrm{Kg}$ para população em geral.

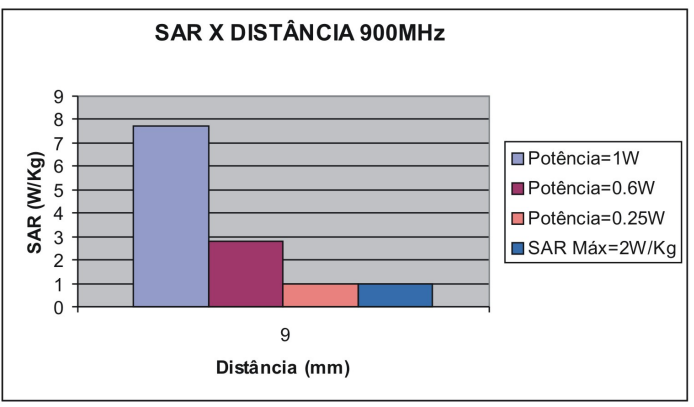

Fig. 4

TAXA DE ABSorÇÃo EsPeCífiCA PARA FAIXA DE $900 \mathrm{MHZ}$

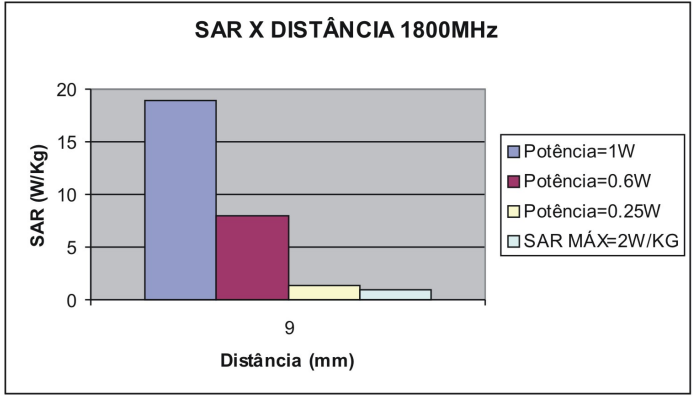

Fig. 5

TAXA DE ABSORÇÃo ESPECÍfICA PARA FAIXA DE $1800 \mathrm{MHZ}$
Notadamente, analisando as figuras (6) e (7), verifica-se que em algumas regiões do cérebro apresentam maior ou menor concentração de energia, que se dá pela presença de atividade do campo elétrico. Observa-se na figura (6) alguns ponto de concentração de energia que indica uma baixa atividade cerebral. Enquanto que na figura (6), verifica-se uma intensa atividade cerebral provocada pelo campo elétrico; de modo que estas atividades estão relacionadas com a taxa de absorção específica(SAR).

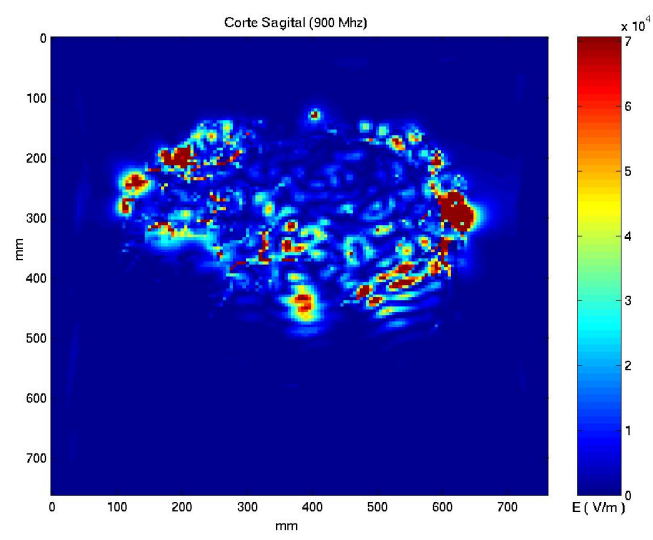

Fig. 6

GRÁFICO DA SAR APÓs 1750 INTERÇõES PARA FAIXA DE 900 MHz

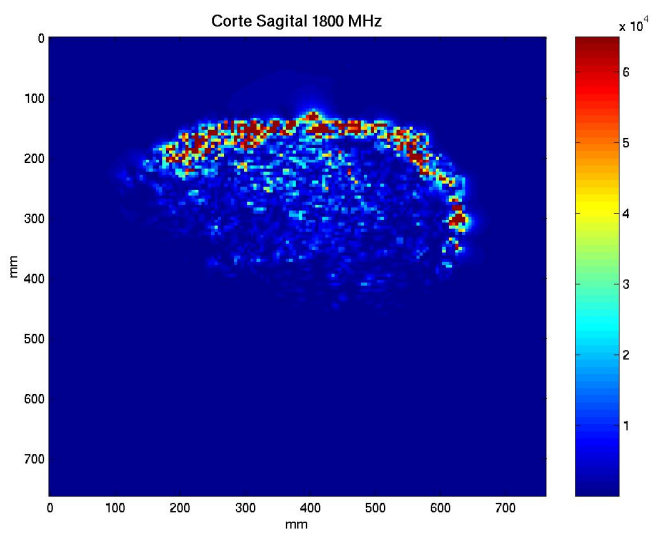

Fig. 7

GRÁFICO DA SAR APÓs 1750 INTERÇõES PARA FAIXA DE 1800 MHz

\section{CONCLUSÃO}

Neste trabalho descreve-se o desenvolvimento de um método numérico LN-FDTD (Local Non Orthogonal Finite Difference Time Domain), que é usado para determinar absorção de energia eletromagnética na região da cabeça humana. Fornece também, um estudo acerca dos principais aspectos do algoritmo para quantificar a taxa de absorção específica (SAR) na cabeça humana. Os resultados comprovam à utilidade do método na capacidade de verificar a precisão e 
a eficiência na solução de problemas, que envolvem materiais não homogêneos. Também indicaram uma influência significativa de vários tipos de tecidos que formam a cabeça humana, e importantes fatores ao interpretar os resultados experimentais, em particular (para uma onda plana, cuja freqüência é de 900 e $1800 \mathrm{MHz}$ ), para uma taxa média do plano sagital da cabeça. Os valores de pico da SAR são significativamente mais elevados para as regiões; olhos e nariz do modelo da cabeça humana heterogênea. Os resultados apresentados para SAR, na potência de $1 \mathrm{~W}$ e $0.6 \mathrm{~W}$ estão acima dos valores estabelecidos pelas normas de segurança; enquanto que o valor de $0.25 \mathrm{~W}$ satisfaz as exigências das agências internacionais como; ICNIRP e ANATEL.

\section{REFERÊNCIAS}

[1] A. A. Salles, "Riscos á saúde provocados pelos telefones celulares", EGATEA, Revista da Escola de Engenharia UFRGS, vol. 24, $\mathrm{n}^{\mathrm{o}}$ 1, $\mathrm{p}$. 7-16,1997.

[2] A. A. Salles,C.Fernandez, M. Bonadiman, "Simulações do Campo Distante e da SAR na Cabeça do Usuário do Telefone Celular para Antenas Convencionais e Planares", In : E. Fontana e A.J.B de Oliveira. [ed]. Anais do X Simpósio Brasileiro de Microondas e Optoeletronica ,SBMO-Recife, vol. 1, p. 347-351,2002.

[3] A. A. Salles,C.Fernandez, M. Bonadiman, "Simulações da SAR na cabeça e antena planares para telefones móveis", Revista Brasileira de Engenharia Biomédica ,vol. 19, nº 2, p. 77-90,2003.

[4] R. Holland, "Finite-Difference solution of Maxwell's equations in generalized nonorthogonal coordinates", IEEE Trans. Nucl. Sci, vol. 6,vol. 6, Dec. 1983.

[5] J. F. Lee, " Modeling Three-Dimensional Discontinuities in waveguides Using Non-orthogonal FDTD Algorithm", IEEE Trans. On Microwave Theory and Techniques, vol. MTT-40, ${ }^{\circ} 2$, feb, 1992.

[6] S. D. Gedney, " An Anisotropic Perfectely Matched Layer-Absorbing Medium for the Truncation of FDTD Lattices", IEEE Trans. Antennas Propagat, vol.19, p. 1630-1639, Dec. 1996.

[7] R. L Mclntosh, R. McKenzie, and A. Carratelli, “ The Numerica Evaluation of a SAR Measurement Phantom at the Telstra Research Laboratories". WARS02 - Workshop on the Applications of Radio Science", Online]Available: http://www.ips.gov.au/IPSHosted/NCRS/wars /wars2002/proceedings/index.

[8] International Commission on Non-ionizing Radiation Protection (ICNIRP), " Guidelines for Limiting Exposure to Time-Varying Electric, Magnetic, and Electromagnetic Fields (up to 300GHz)". Health Physics..Vol.77, No. 4,p. 494-522, Apr. 1998.

[9] AGÊNCIA NACIONAL DE TELECOMUNICAÇÕES - ANATEL, “ Diretrizes para Limitação da Exposição a Campos Elétricos, Magnéticos e Eletromagnéticos Variáveis no Tempo,". Agência Nacional de Telecomunicações.Brasil, Dez. 1999. 